\title{
Performance Analysis of Chromatic Dispersion Compensation of a Chirped Fiber Grating on a Differential Phase-shift-keyed Transmission
}

\author{
Sung Chul Kim* \\ Department of Communication Engineering, Myongji University, Yongin 449-728, Korea
}

(Received January 9, 2009 : revised March 6, 2009 : accepted March 6, 2009)

\begin{abstract}
The properties of the chirped fiber Bragg grating (CFBG) as a chromatic dispersion compensator in differential phase-shift-keyed (DPSK) transmission are analyzed. Comparisons of a performance of a CFBG in between DPSK and On-Off Key (OOK) are shown by simulations using the commercial numerical modeling software, OptSim ${ }^{\mathrm{TM}}$. In the simulation, we compared the performance of the CFBG when they were used in the RZ-OOK 40 Gbps and the RZ-DPSK 40 Gbps transmission. The simulation results show the performance of an overall transmission with a CFBG in DPSK is inferior to the case of OOK, although DPSK generally has a $3 \mathrm{~dB}$ higher SNR (signalto-noise ratio) than OOK.
\end{abstract}

Keywords: Chromatic dispersion, CFBG, DPSK

OCIS codes: (060.0060) Fiber optics and optical communications; (230.0230) Optical devices

\section{INTRODUCTION}

There has been lots of research in the field of optical modulation technologies such as return-to-zero (RZ), nonreturn-to-zero (NRZ), and phase shift keying (PSK). Among them, the commercially used one is NRZ from OC-1 (54 Mbps) to OC-192 (10 Gbps). Nowadays, an optical speed over OC-786 (40 Gbps) in the back-bone area has been attractive due to the vast spread of high speed optical access networks such as PON, FTTH, and HFC. In the domain of OC-768, PSK transmission, especially differential PSK (DPSK), has several advantages over on-off keying (OOK-RZ, NRZ) such as a 3 $\mathrm{dB}$ higher OSNR [1-3]. In the high speed optical transmission, there are a few obstacles to overcome, that is, an optical chromatic dispersion (CD) and a polarization mode dispersion (PMD). The PMD can be avoided by using a recently developed, commercially distributed optical fiber having very low PMD rather than the past conventional optical fiber. To solve CD, a dispersion compensating fiber (DCF) or a chirped fiber Bragg grating (CFBG) can be used to compensate the optical dispersion resulted from the transmission fiber. In DPSK

*Corresponding author: schkim@mju.ac.kr transmission, most research results ever published have used DCFs rather than CFBGs, and there are few researches about the properties of dispersion compensation of CFBGs. The effects of CFBG on the optical transmission system have been researched in the several papers [4-7]. In this paper, the comparison of a CFBG used in the OOK and the DPSK systems in 40 Gbps speed was shown by simulations. The whole simulations are performed by using the well-known commercial optical communication software, OptSim ${ }^{\mathrm{TM}}$.

\section{SYSTEM DESIGN AND SIMULATIONS}

Fig. 1 shows the usual schematic of an optical DPSK transmission system where a CFBG is used as a chromatic dispersion compensating device

Optical pulses from a pulse laser are modulated in the phase domain through a phase modulator using a signal generator. The data encoded optical pulses are transmitted into the optical fiber, suffering from the chromatic dispersion, which results in pulse broadening. Through a CFBG, the distorted optical pulses can be recovered into recognizable optical pulses. In RZOOK system, an optical pulse laser with Gaussian wave- 
form was used [8], and a pseudo random bit sequence (PRBS) generator and an electrical signal generator with an electrical Bessel filter were used to generate random data bit patterns. The optical pulses from the laser were modulated by the optical intensity modulator, transmitted through the transmission fiber, compensated through a CFBG, and finally detected at the optical intensity receiver. In the simulation, optical losses were not included to only consider the dispersion effect, and hence, optical amplifiers were not used. An eyediagram and an eye-closure were calculated in the OptSim $^{\mathrm{TM}}$ automatically.

In RZ-DPSK, the optical pulses from the pulse laser were modulated in phase domain to encode the data bits from the PRBS, and other configurations are similar to those of RZ-OOK except the DPSK receiver. The lengths of the optical fiber in the simulations were 22 $\mathrm{km}$ and $40 \mathrm{~km}$. The parameters for optical simulations were shown in Table 1.

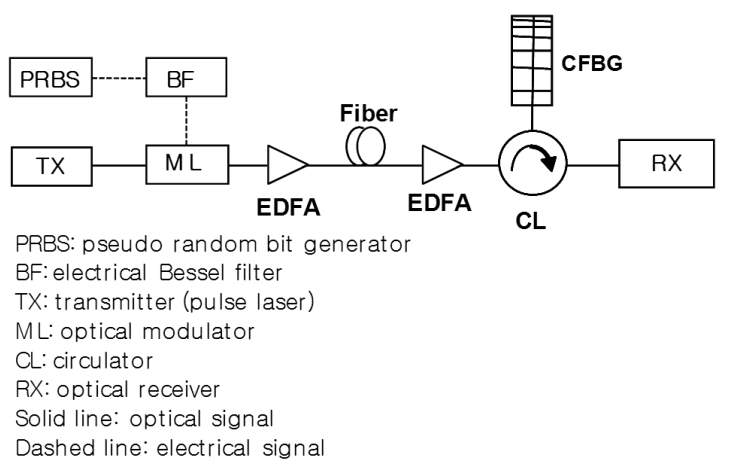

FIG. 1. Schematic of an optical DPSK system with a CFBG as a chromatic dispersion compensator.

TABLE 1. The parameters for optical simulations in both optical modulation schemes.

\begin{tabular}{l|c|c}
\hline \multicolumn{1}{c|}{ Parameters } & Case 1 & Case2 \\
\hline Transmission fiber length & $22 \mathrm{~km}$ & $40 \mathrm{~km}$ \\
\hline Wavelength of light source & $1549 \mathrm{~nm}$ \\
\hline Average power of pulse laser & $1 \mathrm{~mW}$ \\
\hline Bit rate & $40 \mathrm{Gbps}$ \\
\hline $\begin{array}{l}\text { Super Gaussian order of pulse } \\
\text { laser }\end{array}$ & 1 \\
\hline Chirp & $0 \mathrm{rad} / \mathrm{mW}$ \\
\hline FWHM of laser pulse & $16.5 \mathrm{ps}$ \\
\hline Duty ratio of pulse & $33 \%$ \\
\hline samples/bit & 19 \\
\hline Total simulated bits & 1024 \\
\hline Fiber charateristics & Only considering \\
\hline Receiver sensitivity & $--17.9 \mathrm{dBm}$ \\
\hline
\end{tabular}

The wavelength spectra of the CFBG used for the dispersion compensation are shown in Fig. 2. The solid line in Fig. 2 is for an apodized CFBG and the dotted one is for a unapodized CFBG. An apodization, which is to modulate the intensity of an UV (ultra violet) light in the fabrication process of the grating, needs to be done to enhance the properties of the group delay of a CFBG. The modulation profile of a UV light in this paper was a raised cosine waveform, which is generally used in the fabrication of a fiber grating. An unapodized CFBG has a worse group delay response than that of an apodized CFBG. Hence, in the dispersion compensation with a CFBG, the grating should have a good property of a group delay rather than the reflection characteristics of the grating. The reflection spectra of the CFBGs [9] in Fig. 2 were calculated using the MATLAB software.

Fig. 3 shows the group delay properties of the CFBG in Fig. 2. The group delay of the unapodized CFBG has a similar slope with that of the apodized one, but

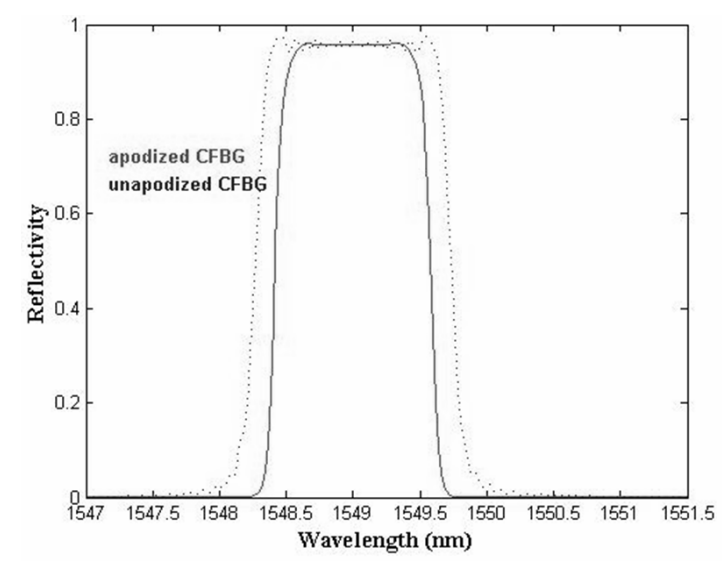

FIG. 2. Reflection spectrum of a CFBG; a solid line is for an apodized grating, a dotted line is for a unapodized grating.

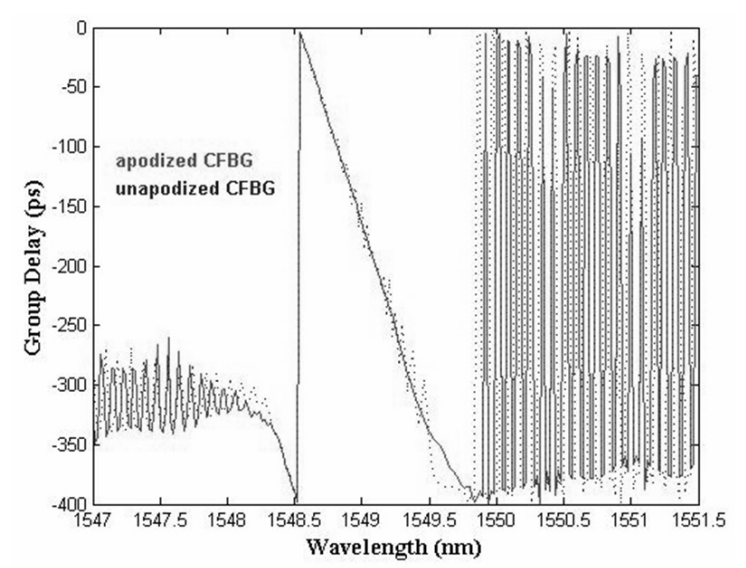

FIG. 3. The group delay properties of the CFBG in Fig. 2; the solid one is for an apodized grating, the dotted line is for an unapodized one. 
it has fast variations through the wavelength. The variation of the group delay is identical to the variation of optical phase because the group delay is calculated from the optical phase. The instability of the optical phase has an effect on the overall performance of the DPSK system. In OOK, the important factors are the reflectivity and the average slope of the group delay, and hence, the minute variation of the group delay is only a minor effect. It is difficult to find the effect of the variation of the group delay in the eye-diagram, but it can be easily recognized in the measure of the eye-closure.

The eye-diagrams for the apodized and unapodized CFBG in OOK and DPSK were calculated and shown in Fig. 4. From the eye-diagrams, an apodized CFBG has a good performance for compensating the chromatic dispersion.

Fig. 5 shows the eye-diagrams of RZ-OOK and RZ-DPSK with a $40 \mathrm{~km}$ transmission fiber and 40Gbps speed when apodized CFBG is used. From the varia-

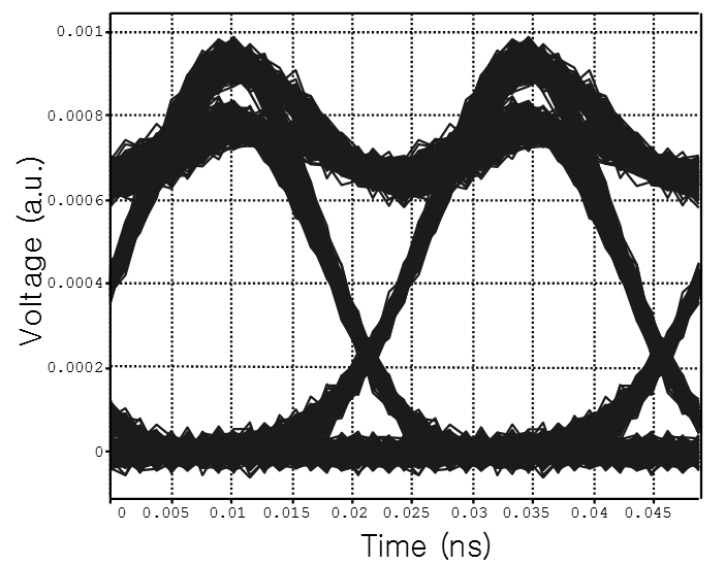

(a)

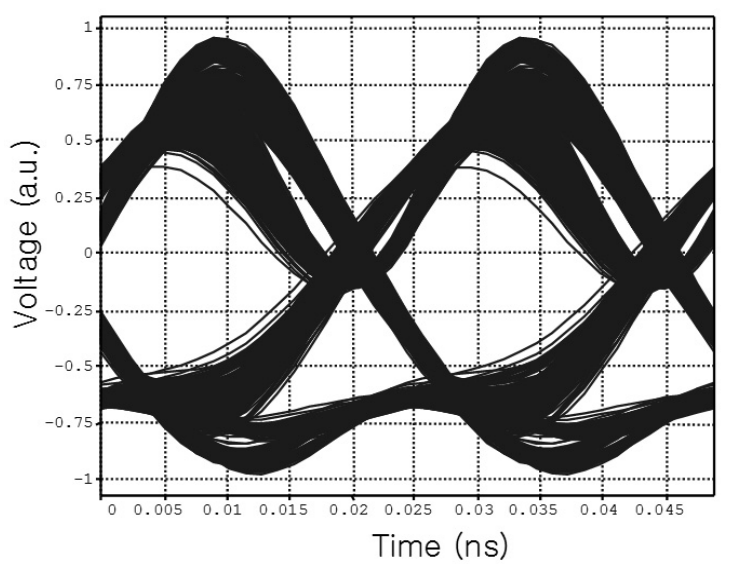

(c) tions of optical phase in the CFBG, the DPSK shows a somewhat closed eye compared to that of OOK.

Fig. 6 shows the comparisons of eye-closures of the RZ-OOK and the RZ-DPSK systems when several CFBGs with various slopes of group delay are used to compensate the chromatic dispersion in $40 \mathrm{Gbps}$ and fiber length of $22 \mathrm{~km}$. Dispersion slope of CFBGs is changed to find the optimized dispersion compensation but they have the same total amount of dispersion. The numbers on the $\mathrm{x}$-axis in Fig. 6 mean the dispersion slope; 0 means an original CFBG with unmodified dispersion slope, the minus number means concave shape of dispersion slope, and the plus number means convex shape of dispersion slope. In Fig. 6, the case of OOK has a lower eye-closure than that of DPSK, although DPSK generally has a $3 \mathrm{~dB}$ higher SNR (signal-to-noise ratio) than OOK. This is caused by the minute phase variation of CFBG, which makes the phase modulated optical signal suffer from higher phase noises through the CFBG. From the calculation

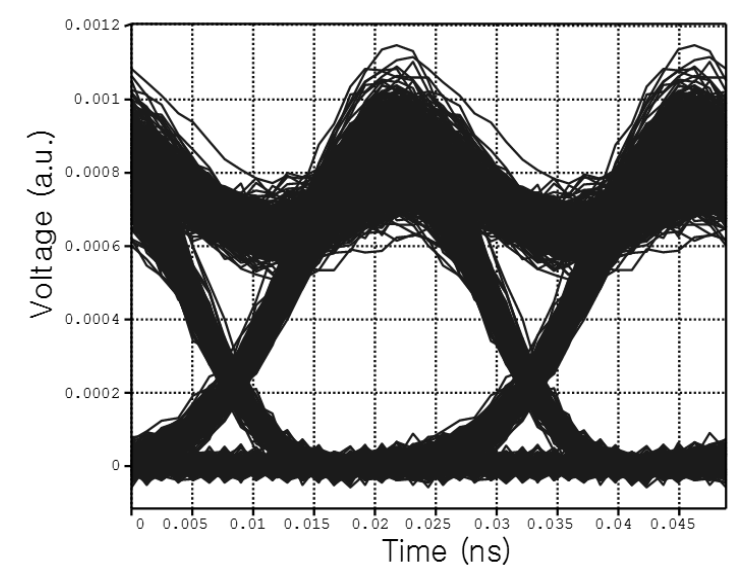

(b)

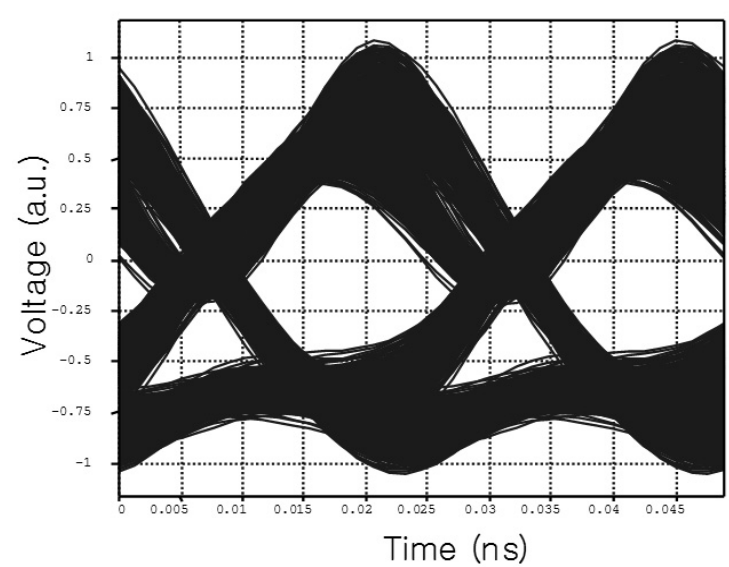

(d)

FIG. 4. The calculated eye-diagrams for (a) 40 Gbps RZ-OOK with an apodized CFBG, (b) 40 Gbps RZ-OOK with a unapodized CFBG, (c) 40 Gbps RZ-DPSK with an apodized CFBG, and (d) 40 Gbps RZ-DPSK with an unapodized CFBG after $22 \mathrm{~km}$ transmission. 


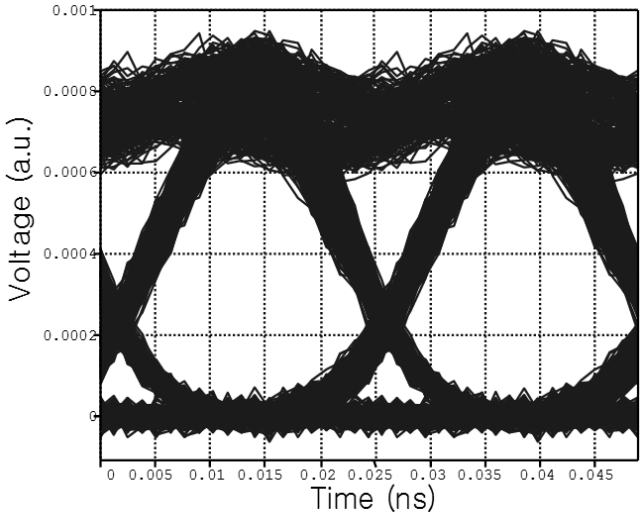

(a)

FIG. 5. Eye-diagrams of (a) 40 Gbps RZ-OOK an

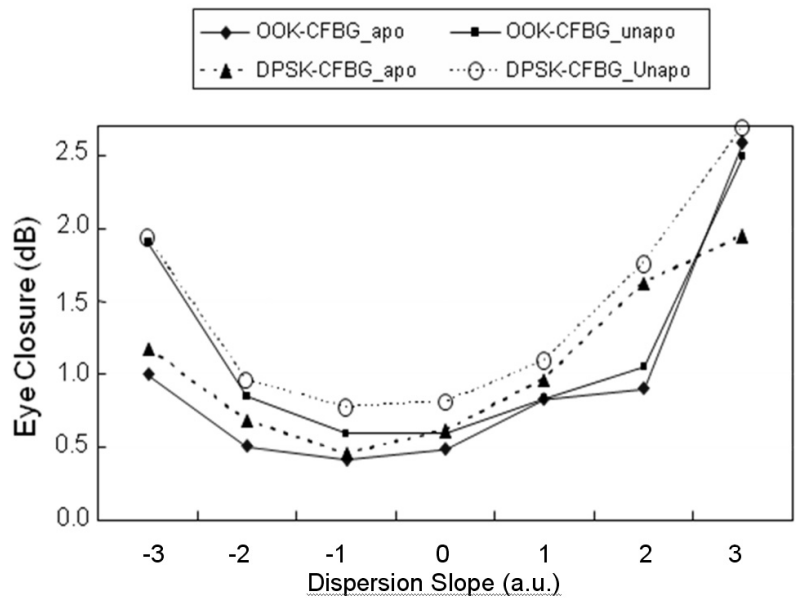

FIG. 6. Comparisons of eye-closures of the RZ-OOK and the RZ-DPSK when a CFBG are used as a dispersion compensator after $40 \mathrm{~km}$ transmission.

results, we conclude that the apodized CFBG has a better performance than the unapodized CFBG in both transmission schemes.

The amount of tolerable group delay ripple of CFBG in DPSK and OOK is calculated and shown in Fig. 7. The quasi-error free limit of Q-factor is near $16 \mathrm{~dB}$, and the RZ-OOK system shows better performance than RZ-DPSK in Fig. 7. From the simulation result, the tolerable group delay ripple in RZ-DPSK was about 115 ps. But this value may be changed according to the changes of system parameters such as optical loss, fiber PMD, fiber transmission length, optical input power, receiver sensitivity, etc.

\section{CONCLUSION}

Conclusively, a CFBG as a dispersion compensating device for the DPSK transmission system has shown

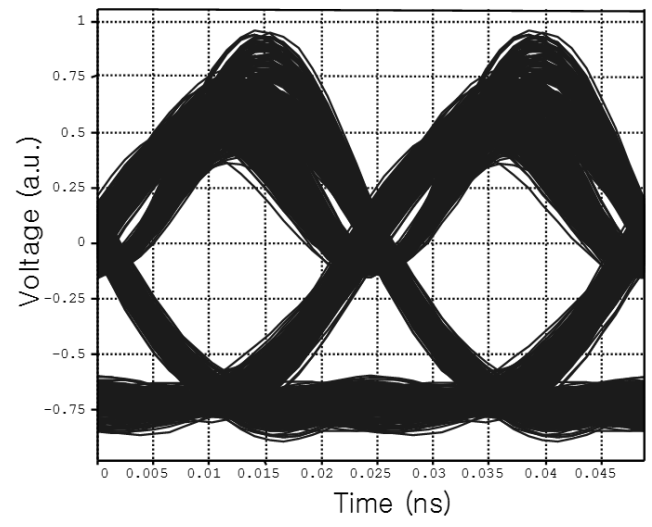

(b)

(b) 40 Gbps RZ-DPSK with $40 \mathrm{~km}$ transmission fiber.

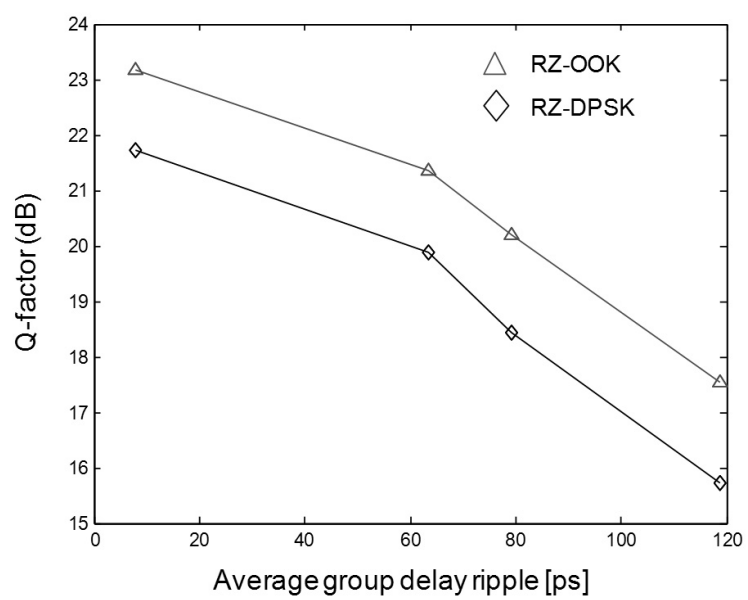

FIG. 7. The effect of group delay ripple of CFBG in view of Q-factor in both transmission systems with 40 $\mathrm{km}$ transmission fiber.

good performance although it is inferior to the OOK., Although the performance of CFBG in view of SNR is inferior to the case of DCF due to the phase variation of the CFBG, a CFBG has several advantages over a DCF such as a small size, a much lower cost, easy handling. In this paper, the comparisons of CFBG when used in RZ-OOK and RZ-DPSK were performed. To compensate the chromatic dispersion in the DPSK transmission system, an apodized CFBG might be used. The simulation results show that RZ-OOK in 40 Gbps has a $0.3 \mathrm{~dB}$ lower eye-closure than that of RZ-DPSK. However, this small value is not high so that a CFBG can be used as a dispersion compensating device in any optical communication system.

\section{ACKNOWLEDGMENT}

This work was supported by the Korea Research 
Foundation Grant funded by the Korean Government (MOEHRD)(KRF-2006-331-D00385)

\section{REFERENCES}

1. A. H. Gnauck and P. J. Winzer, "Optical phase-shift-keyed transmission," IEEE J. Lightwave Tech. 23, 115-130 (2005).

2. H. Kim, "Differential phase shift keying for $10 \mathrm{~Gb} / \mathrm{s}$ and $40 \mathrm{~Gb} / \mathrm{s}$ systems," in Proc. 12th conference on optoelectronics and optical communications (Daegu, May 2005), pp. 189-190.

3. W. Hung, C.-K. Chan, L.-K. Chen, and F. Tong, "An optical network unit for WDM access networks with downstream DPSK and upstream remodulated OOK data using injection-locked FP laser," IEEE Photon. Technol. Lett. 15, 1476-1478 (2003).

4. K. Ennser, M. Ibsen, M. Durkin, M. N. Zervas, and R. I. Laing, "Influence of nonideal chirped fiber Bragg grating characteristics on dispersion cancellation," IEEE Photon. Technol. Lett. 10, 1476-1478 (1998).

5. N. Cheng and C. Cartledge, "Power penalty due to the amplitude and phase response ripple of a dispersion compensating fiber Bragg grating for chirped optical signals," J. Lightwave Technol. 24, 3363-3369 (2006).

6. J. Wang and J. M. Kahn, "Impact of chromatic and polarization-mode-dispersions on DPSK systems using interferometric demodulation and direct detection," J. Lightwave Technol. 22, 362-371 (2004).

7. C. H. Kim, J. Bae, K. Lee, and S. B. Lee, "Performance evaluation of a tunable dispersion compensator based on strain-chirped fiber Bragg grating in a $40 \mathrm{~Gb} / \mathrm{s}$ transmission link," J. Opt. Soc. Korea 12, 244-248 (2008).

8. T. J. Eom, S.-J. Kim, C.-S. Park, and B. H. Lee, "High repetition rate optical pulse multiplication with cascaded long-period fiber gratings," J. Opt. Soc. Korea 8, 29-33 (2004).

9. T. Erdogan, “Fiber grating spectra," J. Lightwave Technol. 15, 1277-1294 (1997). 